PROCEEDINGS OF THE SYMPOSIUM ON

LANGUAGE DESIGN AND PROGRAMMING METHODOLOGY

SYDNEY, 10-11 SEPTEMBER, 1979

\title{
A HUMAN MOVEMENT LANGUAGE FOR COMPUTER ANIMATION
}

\author{
Don Herbison-Evans \\ Basser Department of Computer Science \\ University of Sydney
}

\begin{abstract}
Seven different methods used for specifying human movement are considered: ideographic notation, animation chart, specialised dance languages, natural language, numerical description, demonstration and muscle tension. The prospects of adapting each of them for computer input are discussed. The NUDES language (Numeric Utility Displaying Ellipsoid Solids, version 3) is described and related to this discussion. Current problems with movement specification are also discussed.
\end{abstract}

\section{INTRODUCTION}

From time immemorial humans have sought to represent pictorially their shape and movements. With the advent of computer graphics, there have been a number of attempts to draw human figures with devices controlled by computer. The simple reproduction of outline and tonal value pictures by computer graphic devices allows manipulation of the shape and representation of the figure. Some results have shown great artistic skill, e.g. "Transformations" by Csuri and Shaffer (Franke, 1971), but these types of manipulation do not easily allow natural articulation of the figure.

Attempts to represent all or part of human figures which can articulate have been made for car crash simulation (Bartz, 1973) ergonomic (Fetter, 1964) and choreographic (No11, 1967) studies.

The present study was initiated by the choreographer Phillipa Cullen. She indicated that the general problem of describing human movement in an economic, explicit, unambiquous, easily comprehensible fashion, especially as a linear string of conventional symbols was far from solved. To examine this problem, the seven current methods of describing human movement require analysis.

\section{TYPES OF LANGUAGE}

\subsection{Ideographic Notation}

For several hundred years, dancers and choreographers have realised that the constraints of movement on the parts of a human body have an analogy with the constraints of syntax on language, and have sought a 
language to describe human movement. Hutchinson (1968) reviews 23 notation languages published over the last four centuxies for this purpose, starting with Arbeau (1589). Generaliy these languages are two dimensional and show either a vertical (plan) view of the movements of the figure, e.g. Feulliet (Savage and officer, 1978) or a series of horizontal views of an abbreviated figure (e.g. Zorn, 1905), or both, e.g. Benesh (Causley, 1967). Typically, they have a complex alphabet of special characters whose size, position, orientation and juxtaposition are part of the language. They all suffer from the problem of trying to reduce a multiple four dimensional continuum (space plus time for each joint/limb) onto a two dimensional piece of paper. This can only be done by quantisation of at least two dimensions, and this accounts for the proliferation of symbols, e.g. Laban notation uses 1421 different symbols.

The two main notations in common use are Laban (Hutchinson, 1970) and Benesh (Causley, 1967).

Laban is probably the most widely taught dance notation. It was originated by Rudolf Laban in 1928 in the USA. It has a vertical stave of several columns with time notionaliy running upwards. Different columns of the stave are reserved for movements of the different parts of the body: the legs, arms, torso, head, etc. Motions for each part are maxked for those time periods in which they occur, i.e. time is treated in a continuous analog fashion. various symbols placed on the stave represent the various directions and amounts of movement. Thus space is quantised. There are 27 main directions, and intermediate directions are specified by additional symbols. Laban notation is propagated by the Dance Notation Bureau in New York, who maintain a library of the scores of many dances, and ensure its standardised application.

Benesh notation is probably the most widely used notation in professional dancing. It was devised by Joan and Rudolf Benesh in the UK in 1947. It is arawn on a horizontal musical type of stave. Time runs left to right as in music, which facilitates synchronisation of movement with the music, as the music can be placed above the notation. At appropriate intervals along the stave are drawn symbolic pictures of the figure and its movements since the last picture. The bottom line of the stave represents ground level. Thus positions in the plane of the figure are recorded in an analog fashion but time is quantised. The third spatial dimension is communicated by the foreshortening of the parts of the body, up to an ambiguity about whether they are in front of or behind the plane of the figure. By using special symbols for these two possibilities, Benesh effectively manages to show analog representations of all three spatial dimensions on a two dimensional piece of paper.

Benesh notation is propagated by the Institute of Choreology in London, who also maintain a library of the scores of many dances. Many professional ballet companies now have staff choreologists who record and interpret the dances at rehearsals including the Royal Ballet at Covent Garden, and also the Australian Ballet.

Both Benesh and Laban require a three year learning course although shorter courses are offered for those only wishing to read 
the notation. Neither notation can be written in real time, and in both cases the preparation of the final score is an arduous task.

\subsection{Animation Chart}

This is used by makers of animated cartoons. The cartoon frames are generated by a series of overlapping transparent celluloid sheets with parts of bodies or scenes painted on them. These are called cels. Every cel is given a separate identifier. The animation chart has a set of column, one column for each part of the body or scene which moves. Across these columns are rows representing each frame of the film. Specifications are written in each row of the cels that are in use for that frame. For example in cheapex cartoons the same set of cels may be used several times to show the legs moving in a walk cycle. The set of cels would be written into its column at the several appropriate places in the chart.

The animation chart has some similarities to Laban notation, with time running vertically and body parts spaced horizontally.

\subsection{Specialised Dance Language}

For several styles of dancing, specialised technical terms have evolved that describe the movements and positions of that style, e.g. square dancing, ballroom dancing (Moore, 1936), classical ballet (Vaganova, 1969). A sequence of these terms serves to describe fully a dance of the appropriate style.

\subsection{Natural Language}

To describe human movement, a variety of nouns, adjectives, verbs and adverbs are available which can describe the spatial disposition and movements of the body. It has been estimated that $20 \%$ of the words of English are concerned with spatial position (Ha11, 1969).

Many words describe movement by specifying contact with some other part of the body or external surface, e.g. grasp, kick, kiss. Even more powerful are the words which describe bipedal locomotion: walk, run, skip, strut, tiptoe, shuffle, sneak (Blair, 1949).

\subsection{Numerical Description}

By this is meant a prescription of the positions and orientation angles of the parts of the body in every frame of an animated sequence. One of the published ideographic dance notations approaches this form (Eshkol and Wachmann, 1958).

\subsection{Demonstration}

This is the usual method employed in professional dance for communication of human movement (Tomkins, 1970). When a company adds a new dance to its repertoire, either the choreographer or a principal 
dancer from a previous production elsewhere is imported to teach it to the company. Each dancer is then shown the movements usually in segments of a few seconds duration. These must be remembered and concatenated by each dancer. A full ballet lasting over an hour involving perhaps 30 or more dancers can require rehearsal times of several months for this process.

\subsection{Muscle Tension}

This is the body's own language. Given the masses and moments of inertia of the parts of the body, and the distances between joints and points of insertion of the muscles on the skeleton, the forces and couples generated by the muscles serve to determine completely the motions of the body in accordance with Newton's laws of motion (Wells, 1971). Hence movements can be notionally specified by a sequence of muscle tensions.

\section{Computerisation}

In principle any of the seven types of language can be adapted and used to communicate with a computer which has as its output a graphic display of an animated human figure. Each method has advantages and drawbacks.

Several attempts have been made to use some of the ideographic notations.

A subset of Massine notation has been successfully implemented and used in dance teaching (Savage and officer, 1978). Regrettably the computer system on which it was implemented has been superseded and the software has proved impossible to transfer to the replacement hardware. The system allowed the interactive generation and editing of a small score and it then played this back as a stick figure performing a dance. The system was confined to movements on the spot: locomotion was not implemented.

A substantially complete editor has been implemented for Laban notation (Brown et al., 1978). It uses a storage tube display which limits selective erasure and makes it somewhat difficult to use. The system is being rewritten for a refresh display, and the group also plans to drive a figure with the stored score.

A project of this type was also proposed for Benesh notation (Mendo, 1975). This involved the input of existing scores by tracing then with a digitiser. They were then to be analysed and a dancing figure generated from them. Although the system has been designed down to the data representation and analysis stage, it has not yet been implemented.

Al1 three above systems use the two dimensions of the screen/ page. This contrasts with the normal mode of computer input which is a character string: one dimensional and quantised. Attempts have been made to transiterate ideographic notations into this form. 
tion but did not translate it (Keen, 1973). A complete transliteration and translation has subsequently been implemented (Calvert and Chapman, 1978). Only a small subset of Laban was implemented, but this has been made to drive a gesturing stick figure. Again, locomotion was not translated.

Similarly a subset of Benesh has been transliterated and translated and used to drive a multiellipsoid figure (McNair, 1979). This was outstanding in that locomotion was included. However only the general position signs of the body and limbs were implemented. The movement signs and more detailed symbols were not included.

The complexity of extending the systems to include both locomotion and movement details has so far proved a barrier for all the ideographically based systems.

The animation chart has been implemented for the computer generation of cartoons (Catmull, 1978), but has not proved very successful. Nevertheless, the concept of applying a number of sets of actions over a series of possibly overlapping quantised time periods is valuable. It gives very flexible time control of complex actions.

An attempt is being made to translate the specialised terms of classical ballet (Hunt, 1978). The interfacing of adjacent movements, which is normally left to a dancer's common sense, is a serious but not insuperable problem. Certain aspects of specialised language map well into a computer language. Each technical term can be viewed as a subroutine/macro call. This deals well with complex action sequences but gives only simple timing control. A more serious drawback is the limited types of movement that can be produced by a system that is tied to a specialised style of dance.

This can be overcome by using full natural language which gives useful facilities such as command verbs, body part names, adverbs for acceleration control and named sequences of actions. The disadvantage of natural language is its lack of precision in the specification of timing and position, except for the case when movement ends in touching a surface.

Demonstration has been used in the past for communication with computers. This has taken several forms:

A. A human wearing an exoskeleton with potentiometers at the joints. Readings are fed back to the computer and stored (Toscas, 1971). This is useful for generating specific movement sequences, but is rather cumbersome for general movement synthesis.

B. Cine films of moving humans taken from different points of view have been measured frame by frame and the measurements used to reconstruct a moving stick figure (Shigeru ohe, 1969).

C. A virtual puppet visible on a screen has been provided which can be manipulated by conventional interactive devices (Withrow, 1970). The movements are recorded and played back as required. The figure used was a stick figure, and only gesturing, not locomotion was implemented. 


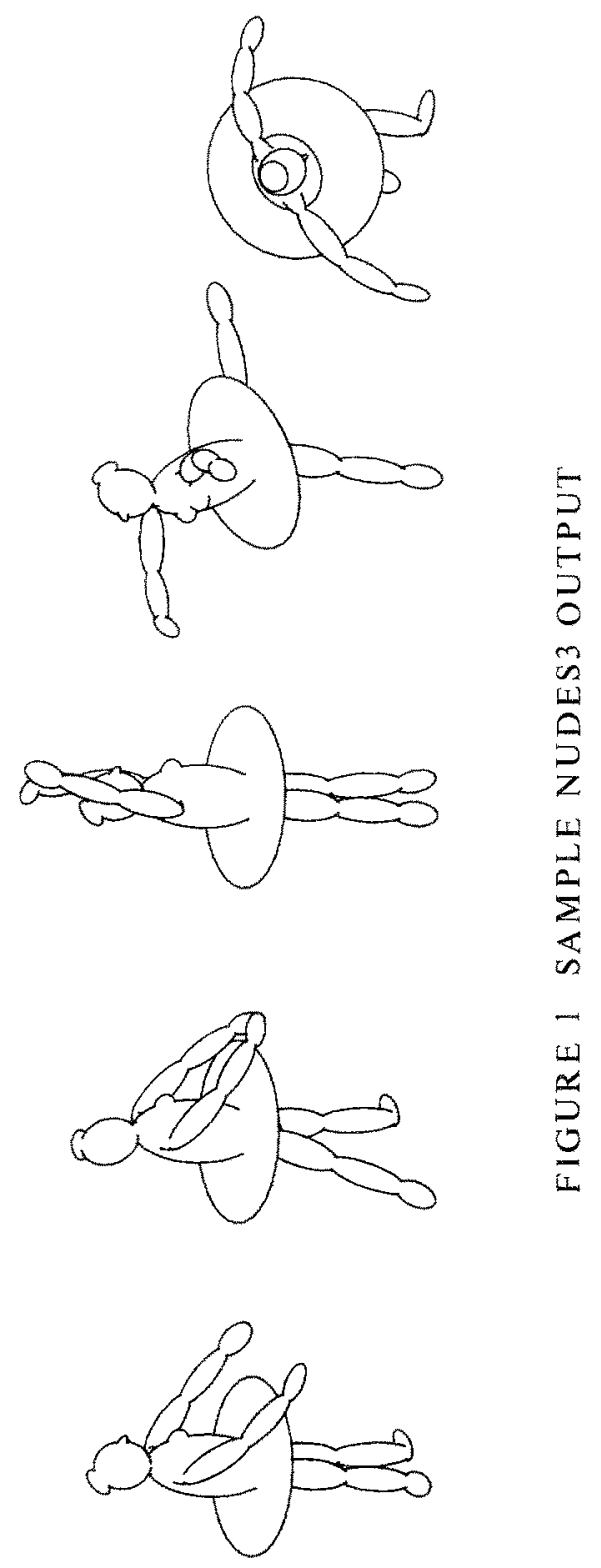


This last technique is potentially very useful to a choreographer, and the problems of extending it are discussed later in the paper.

Numerical description was tried by the author at an early stage (Herbison-Evans, 1974). It is a tedious error-prone method of programming anything substantial. It is however a necessary intermediate language in the translation of more sophisticated human movement languages.

Muscle tensions may be useful for kinesic and physiological studies but is a difficult mode for controlling figure activity. Some indication of the difficulty of using this language for figure control may be gleaned from an observation of the time required to generate, say, a sequence for walking by a normal human naturally, namely about two years.

\section{NUDES 3}

This Numeric Utility Displaying Ellipsoid Solids (version 3 ) is a procedural language that attempts to combine the generality of natural language with the temporal control of the animation chart and the precision of numerical language. Like natural language the user gives and uses names for the figures, body parts, joints and action sequences. Also a vocabulary of words is predefined for use in commands and acceleration control. It is like an animation chart in that every action is specified by a set of frames in the resulting film. These are identified by their frame numbers. It is numerical in that the sizes of the body parts (ellipsoids), the relative positions of the joints, and the displacements and rotations in the actions must all be specified numerically.

\subsection{Implementation}

The NUDES3 language program is processed by a suite of five programs. Four are written in ANSI FORTRAN IV. The fifth is in IMLAC assembler. The first converts the NUDES3 program to a numerical sequence of three dimensional positions and orientations. The second projects these into two dimensions and works out which outline arcs are obscured. The third draws these on a printer page or calcomp plotter. The fourth compresses the two-dimensional data numbers to two digit base sixty numbers which are implemented as ASCII characters for transmission down a low speed data line to an IMLAC PDS4. The fifth generates from this compressed data a 16 movie using a computer controlled stopped frame animation camera attached to the PDS4.

A sample plotter output is shown in Figure 1.

The current limitations of the suite are a maximum of 41 ellipsoids and 300 actions.

\subsection{Layout}

All commands have a free format with the space character used as 
a separator between words, values, etc. An asterisk is used as a signal to ignore the rest of a line/card. This allows commentary to be included among the commands.

Each action keyword has an expected set of parameters following it. The order of these parameters is predefined, and hence the position of a parameter determines its type.

Variables may be used in place of values or in place of the names of figures, joints, ellipsoids, axes, or subroutines.

All keywords and names may be arbitrarily abbreviated by using only the first one or more characters. It is however unwise to abbreviate to the point of ambiguity with other names or keywords. Names may be any length but only the first six characters are used by the compiler. It is also unwise to use a name which is the same as the beginning of any other name or keyword.

A NUDES 3 program is divided into two parts: a set of declarations and a set of action specifications.

\subsection{Declarations}

Each figure must be declared by using the keyword FIGURE followed by a name for the figure, then the number of body parts, and then a list of the names of those parts.

The actual implementation of the drawing routines assumes that the body parts are ellipsoids, so each of these parts must then be defined by using the keyword ELIIPSOID followed by its name and by the lengths of the $x, y$, and $z$ semi-axes.

Each joint must be defined by using the keyword JoINT followed by its name and the names of the two ellipsoids that it joins. Each of these ellipsoid names must be followed by the relative $x, y, z$ coordinates of the joint with respect to the ellipsoid centre. The ellipsoids may only be simply connected by the joints.

The names of all variables and subroutines must be declared using the VARIABLE and DECSUB keywords respectively. In each case the keyword must be followed by the number of names in that declaration and then by the names themselves. Any number of VARIABLE and DECSUB declaration statements may be used.

The various declarations can occur in any oxder provided only that the ellipsoid names are declared as part of a figure before being dimensioned or jointed.

\subsection{Actions}

Action commands begin with a keyword and two frame numbers. The keyword is one of: REPEAT, LINEAR, QUADRATIC, ACCELERATE, Or DECELERATE, depending on how the action is required to be distributed over the specified frame sequence. 
Actions can be specified to be limited to one figure or to apply to all figures. The available actions are:

GROALL, GROFIG: these cause all dimensions of all or of one of the figures to be multiplied by a scaling factor.

MVALTO, MVALBY, MVFGTO, MVFGBY cause absolute or relative translation of all or of one of the figures by the amounts specified in the command in the $x, y, z$ directions. Absolute movements are specified by nominating an ellipsoid whose centre is moved to the point specified.

GRNDAL, GRNDFG: these cause one or all of the figures to be moved vertically (in the y direction) so that the lowest point of the figure(s) just touches $y=0$.

The coordinate axes used are left handed: $x$ is from left to right; $y$ is up; $z$ is away from the observer.

RTALPT, RTFGPT, RTALEL, RTFGEL cause rotation about a specified point or about the centre of a nominated ellipsoid of all or one of the figures by a specified angle about a specified axis $(x, y$, or $z)$.

BENDBY, BENDTO cause articulation of the figure containing a nominated joint at that joint, moving a nominated ellipsoid. The bend can be specified as relative by using the BENDBY command, or absolute using the BENDTO. For a relative bend the rotation of the moving subfigure (attached to the moving ellipsoid) is specified by an angle and axis fixed in a nominated reference ellipsoid. For an absolute bend, the required Eulerian angles of the nominated ellipsoid are specified with respect to a nominated reference ellipsoid.

In both the rotation and the bend commands, angles are specified as positive if they are anticlockwise when viewed from the reference origin along the axis of rotation. They are specified in degrees.

SUBROUTINE, END, and CALL allow a sequence of actions to be named, and for that sequence to be called from elsewhere. The minimum and maximum frame numbers used inside the subroutine are purely formal, and are offset to match the actual frame numbers in the call of the subroutine.

SET assigns a value to a variable for every frame between the specified pair of frame numbers. The value may be that of another variable, which can be that of another variable, etc. to any depth up to the maximum number of allowed variables. Any variable may hold a value that is a specification of a displacement, angle, growth factor, ellipsoid, figure, joint, or subroutine. The user is encouraged to use types in a consistent manner.

VIEW and SPEED allow checking of animated sequence without generating all of it. VIEW allows a subsequence of frames to be generated. SPEED allows extra frames to be interpolated or all but every nth frame to be deleted. Both may be used once only in a 
NUDES 3 program. If both are used, then the frame numbers in the VIEW command refer to those numbers before the effect of the SPEED cormand is applied. formal.

The frame with the lowest frame number is not drawn, and is purely

\section{CURRENT PROBLEMS}

A major problem is the specification of rotations in the rotation and bend commands. The NUDES3 numerical method is very error prone, particulaxly the specification of the sign of the required rotation angle. The BENDTO command is also a difficult concept to understand and use. Possibly the commands should be altered to use verbal descriptions of the signs of the angles and of the required axes, with words like "up", "down", "left", "right", "forward", "back". The difficulty with this is that in naming a direction relative to some other part of the body, if that part is already oriented abnormally, then the description will again be very unnatural, e.g. what direction is "forward" for the arms when the torso is twisted 90 degrees at the waist?

Another major problem is how to specify and to determine the complex motions required to bring one part of a figure into some specified position by moving other parts, e.g. move the hand to a specified position by bending the arm at the shoulder. This is exacerbated by the need to forbid parts of the body to interpenetrate and also by the limited angular movement available at the joints.

An extension of this problem is the general need for commands for motion until some part of the figure contacts a specified surface. Such contacts are essential for serious dance work, for example when two figures are dancing together. Even in natural language the importance of contact can be understood, e.g. in such a film classic as "Holstering his gun, he leapt onto his horse, tipped his hat, and galloped of into the sunset". Calculating such contacts appears to require timeprohibitive iterative techniques. An analytic algorithm for finding the rotation that will cause two nominated ellipsoids to touch is required.

One overall basic problem with NUDES 3 however is that it is designed for working in a batch environment. What is needed is not a programing language but an interactive command system.

A choreographer or animator needs a set of three dimensional puppets each with appropriate store and recall facilities for movement sequences, plus a set of simple manipulation commands to generate and edit such sequences. Then directions of rotation and the generation of contact can be determined visually.

The puppets must be notionally solid. The stick figures used in most systems to date give some three dimensional cues by foreshortening but do not resolve the in front/behind ambiquity. Only apparently solid figures can do this by the removal of hidden lines. Doing this in still somewhat expensive (Watkins, 1970; Herbison-Evans, 1979). 


\section{REFERENCES}

ARBEAU, T. (1589): "Orchesographie". Jehan des Preyz, Langres.

BARTZ, J. (1973): "Modeling People in Car Crashes". New Sceintist, 23 Aug. 1973, p.447.

BLAIR, P. [c 1949]: "Animation". Foster Art Service Inc., Calif., p.24.

BROWN, M., SMOLIAR, R.W., and WEBBER, L. (1978): "Preparing Dance Notation scores with a Computer". Computers and Graphics, Vol.3, pp.1-7.

CALVERT, T.W., and CHAPMAN, J. (1978): "Notation of Movement with Computer Assistance". Proc. Annual Conf. A.C.M., pp.731-736.

CATMULL, E. (1978): "The Problems of Computer Assisted Animation". Computer Graphics, Vol.12, No.3, pp.348-353.

CAUSLEY, M. (1967): "An Introduction to Berest Movement Notation". Man Parrish, London.

ESHKOL, N., and WACHMANN, A. (1958): "Movement Notation". Weidenfeld and Nicolson, London.

FETTER, W.A. (1964): "Computer Graphics in Communication". McGrawHill, New York.

FRANKE, H.W. (1971): "Computer Graphics Computer Art". Phaidon, London, p.32.

HALL, E.T. (1969): "The Hidden Dimension". Anchor, New York, p.93.

HERBISON-EVANS, D. (1974): "Animated Cartoons by Computers Using El1ipsoids", Proc. 6th Australian Comp. Conf., pp.811-823.

HERBISON-EVANS, D. (1979): "Algorithms for Real Time Animation of Drawings of the Human Figure with Hidden Iines Omitted". Sydney University: Basser Dept. of Computer Science, Technical Report 148.

HUNT, F. (1978): Department of Computer Science, University of Utah, Private Communication.

HUTCHINSON, A. (1968): "Experiences of Dance Notations". Dance Magazine, pp. 304-308.

HUTCHINSON, A. (1970): "Labanotation", Oxford University Press, London, Second Edition. 
KEEN, J. (1973): "Movement". Sydney University, Basser Dept. of Computer Science, Honours Report.

MCNAIR, B. (1979): "Benesh Movement Language", Sydney University, Basser Dept. of Computer Science, M. Sc. Reprot.

MENDO, G., (1975): "Computer Choreology Project", Institute of Choreology, London.

MOORE, A. (1936): "Modern Ball room Dancing". Ritman, London.

NOLI, A.M. (1967): "Choreography \& Computers".

SAVAGE, G.J., and OFFICER, J.M. (1978): "Coreo: an Interactive Computer Model fox Dance". Internat. J. Man-Machine Studies, Vol.I0.

SHIGERU OHE (1969): "Computer Animation", Film, Moore School of Elec. Eng., University of Pennsylvania.

TOMKINS, C. (1970): "An Appetite for Notion" in "The Dance Experience", Edited by Nadel M.H., and Nadel C.G., Praegar, New York, p.257.

TOSCAS, G. (1971); "Instant Animation from a Magic Box". International Photographer, February.

VAGANONA, A. (1969): "Basic Principles of Classical Ballet". Dover, New York.

WATKINS, G.S. (1970): "A Real Time Visible Surface Algorithm", Dept. of Computer Science, University of Utah, Report UTEC-CSC-70-101.

WELLS, K.F. (1971): "Kinesiology". Fifth Edition, Sanders, Philadelphia.

WITHROW, C. (1970): "A Dynamic Model for Computer Aided Choreography", Dept. of Computex Science, University of Utah, Report UTEC-CSC$70-103$.

ZORN, F.A. (1905): "Grammar of the Art of Dancing". Franklin, New York. 